\title{
Riqueza estacional y estructura de la comunidad de hormigas epígeas en fragmentos de bosque de sabanas inundables, Orinoquia Colombiana
}

\author{
Juan Carlos Agudelo-Martínez ${ }^{1} \&$ Néstor Pérez-Buitrago ${ }^{1}$ \\ 1. Grupo de Investigación en Ciencias de la Orinoquia (GICO), Universidad Nacional de Colombia, Sede Orinoquia \\ Kilómetro 9 vía Arauca-Caño Limón; jcagudelo@gmail.com,nfperezb@unal.edu.co
}

\author{
Recibido 03-II-2020. Corregido 27-VI-2020. Aceptado 01-VII-2020.
}

\begin{abstract}
Seasonal richness and structure of the epigeal ants (Hymenoptera: Formicidae) community in forest fragments of the Colombian Orinoquian flooded savanna. Introduction: Ants are one of the most abundant and diverse taxonomic groups. Their ecological fidelity allows them to carry out indispensable roles for the adequate functioning of terrestrial ecosystems. Objective: Evaluate seasonal biodiversity dynamics (richness and abundance) of ant species associated with fragments of forest and the surrounding floodplain savanna at the municipality of Arauca, Colombia. Methods: Ants were collected using three complementary techniques (pitfall, Winkler sac and direct collect), in eight forest fragments where linear transects with seven sampling points were installed. Non-parametric estimators and the complementarity index were used to describe the ant community structure. The climatic seasons (dry and rainy) and environments (floodplain, edge, and forest) were compared using species richness and abundance. The relationship of ant species by each sampling points was evaluated with a non-metric multidimensional scaling analysis (NMDS) and the forest fragment attributes were related to species richness through a canonical correspondence analysis (CCA). Results: We found 30496 specimens corresponding to 104 species, 37 genera, and eight subfamilies. The best-represented genera were: Pheidole, Camponotus, Solenopsis, and Dolichoderus. The richness nonparametric estimators showed that the ant community oscillates between 107 and 119 species, no differences were detected in species richness and abundance in terms of the climatic seasons (dry and rainy). There were differences in species richness between floodplain with both edge and forest environments. Ant richness fragments of the forest was related by fragment variables such as area, perimeter, fractal dimension, and compactness index. Conclusions: No differences in species richness and abundance were detected between the dry and rainy seasons, which suggests that the species are permanent residents of the study area. There must be mechanisms that allow these ant species to cope with the rainy season. There was a direct relationship between different forest fragment characteristics and ant species richness. This work expands the knowledge of the ant species present in the north of the Colombian Orinoquia.
\end{abstract}

Key words: landscape; Formicidae; ant biodiversity; Arauca; species richness pattern; seasonality.

Agudelo-Martínez, J.C. \& Pérez-Buitrago, N. (2020). Riqueza estacional y estructura de la comunidad de hormigas epígeas en fragmentos de bosque de sabanas inundables, Orinoquia Colombiana. Revista de Biología Tropical, 68(3), 947-958.

La biodiversidad es un elemento fundamental para el desarrollo en planes de conservación y el uso sustentable de los recursos naturales, por esta razón su pérdida es un tema recurrente en la literatura científica (Myers, 1996). En Colombia, este problema cobra especial importancia teniendo en cuenta que es un país "megadiverso" y de alto grado o nivel de endemismo (Rangel-Ch, 2005; Solari et al., 2013), pero con una cobertura de investigación diferencial entre las diferentes regiones del país. Esto se evidencia en el escaso número de publicaciones científicas sobre biodiversidad en regiones como el Pacífico y la Orinoquia 
(Arbeláez-Cortés, 2013), lo que es atribuido a factores como el aislamiento geográfico, el orden público y la poca presencia de instituciones capaces de liderar procesos de investigación (Agudelo \& Pérez-Buitrago, 2015). Esa situación está en contravía si se tiene en cuenta que la Orinoquia colombiana es considerada por el Fondo Mundial para la Conservación como uno de los ocho ecosistemas estratégicos para la humanidad por su alta riqueza en humedales y biodiversidad (Correa, Ruiz \& Arévalo, 2005). Por esta razón, es prioritario generar iniciativas para subsanar la carencia de información acerca de la biodiversidad en esta región, más si se tiene en cuenta que el estado colombiano la percibe como una despensa de recursos renovables y no renovables, lo cual aumenta el riesgo de transformaciones drásticas de sus ecosistemas en las décadas venideras.

Una estrategia para estudiar la biodiversidad de ecosistemas en grandes extensiones como la Orinoquia colombiana es acudir a la información asociada a especímenes depositados en colecciones biológicas, los cuales funcionan como registros históricos que pueden permitir detectar cambios en las comunidades biológicas como consecuencia de acciones antropogénicas o eventos naturales (Gropp, 2006). Sin embargo, esta aproximación tiene corto alcance para la Orinoquia colombiana debido a la baja representatividad de especímenes de esta región en colecciones biológicas, impidiendo identificar cambios en patrones temporales o espaciales de la biodiversidad. Otra alternativa es el uso de inventarios rápidos que permiten generar conocimiento base acerca de la biodiversidad en áreas poco estudiadas e identificar los efectos de factores que podrían impactar los ecosistemas (i.e. deforestación, erosión, especies invasoras o contaminación) (Wike, Martin, Paller, \& Nelson, 2010). El desarrollo de este tipo de inventarios en áreas representativas de menor tamaño permite la medición de la biodiversidad de forma más eficiente con la posibilidad cautelosa de hacer inferencias a escalas mayores para entender el funcionamiento de los ecosistemas (Vanegas, 2010).
El desarrollo de inventarios rápidos usa organismos como las hormigas (Hymenoptera: Formicidade), puesto que es uno de los grupos más importantes en cualquier ecosistema y permiten la evaluación de impactos causados por disturbios naturales o antropogénicos (Baccaro, Rocha, Aguila, Schietti, \& Emilio, 2013; Falcão, Dáttilo, \& Izzo, 2015; Gerlach, Samways, \& Pryke, 2013; Mcgeoch, 1998). Este taxón reúne características como: alta abundancia (Folgarati, 1998; Lozano-Zambrano, UlloaChacón \& Armbrecht, 2009; Underwood \& Fisher, 2006), fidelidad ecológica (Agosti, Majer, Alonso \& Schultz, 2000; Fernández, 2003; Majer, 1983) y taxonomía relativamente estable que les permite ser usadas como indicadores ecológicos y de biodiversidad en sistemas naturales y perturbados (Andersen, Fisher, Hoffmann, Read \& Richards, 2004; Andersen $\&$ Majer, 2004). Teniendo en cuenta la necesidad urgente de estudiar la biodiversidad de la región de la Orinoquia Colombiana, este estudio tiene como objetivo documentar la dinámica temporal asociada al régimen climático de la biodiversidad (riqueza y abundancia) de especies de hormigas asociadas a fragmentos de bosque en la sabana inundable del municipio de Arauca (Colombia).

\section{MATERIALES Y MÉTODOS}

Área de estudio: La recolección de especímenes se llevó a cabo en ocho fragmentos de bosque con áreas entre los 0.51 y 200 ha y distancias entre 6.8 y $14.1 \mathrm{~km}$ del casco urbano de la ciudad de Arauca. Todos los fragmentos están rodeados por sabana inundable en la zona rural del municipio de Arauca dentro del polígono delimitado por las siguientes coordenadas: $7^{\circ} 4^{\prime} 1.21^{\prime \prime} \mathrm{N} \& 7^{\circ} 49^{\prime} 20.14^{\prime \prime} \mathrm{W}$ $7^{\circ} 4>3.65$ » N \& 70³9’18.68” W - 6 $57>50.98$ » $\mathrm{N} \&$ \& 70³9'19.62" W - 6 57 \% 49.70 » N \& $70^{\circ} 49^{\prime} 17.52^{\prime}$ W. La zona de estudio se caracteriza por un régimen de precipitación unimodal con un periodo de lluvias de ocho meses entre abril y noviembre y un periodo seco de cuatro meses entre diciembre y marzo (Rippstein, Amézquita, Escobar, \& Grollier, 2001). El 
ecosistema predominante es de sabana inundable con presencia de bosques de galería y fragmentos de bosque aislados (Minorta-Cely \& Rangel-Ch, 2014).

Trabajo de campo: Se realizaron dos muestreos, uno en temporada seca entre marzo y abril y el segundo en temporada de lluvias entre septiembre y noviembre 2014. En cada uno de los ocho bosques se instaló un transecto de $190 \mathrm{~m}$ perpendicular al borde del fragmento abarcando ecosistemas de sabana inundable y bosque. Cada transecto consistió de siete estaciones de $10 \mathrm{~m}^{2}$ separadas por una distancia de $20 \mathrm{~m}$ (tres estaciones en la sabana inundable, una en el borde del fragmento de bosque y tres dentro del fragmento de bosque). En cada estación se usó una modificación del protocolo ALL (Ants of the Leaf Litter Proto$\mathrm{col}$ ) que consistió en la implementación de tres técnicas complementarias de recolección: una trampa de caída instalada por 24 horas en el centro de cada estación; una muestra de $1 \mathrm{~m}^{2} \mathrm{de}$ hojarasca por estación procesada en saco mini Winkler durante 48 horas y recolección directa de especímenes en cada una de las estaciones fue llevada a cabo por dos personas durante 10 minutos usando pinzas entomológicas y aspiradores bucales (Agosti et al., 2000).

Laboratorio: Las muestras recolectadas fueron trasladadas y depositadas en la Colección Entomológica de la Universidad Nacional de Colombia sede Orinoquia (CEO) donde se realizó la separación, limpieza, almacenamiento, etiquetado e identificación de los especímenes hasta el nivel taxonómico más explícito posible, el conjunto de datos puede ser consultado en el GBIF y SiB-Colombia (doi:10.15472/vp8v42). Para la identificación a nivel de subfamilia y género se usaron las claves taxonómicas de Bolton (1994) y Palacio \& Fernández (2003). Para la identificación a nivel de especie se usaron las claves taxonómicas de Brown (1976, 1978), Jiménez, Fernández, Arias, \& Lozano-Zambrano (2007) y Mackay \& Mackay (2010).
Análisis de datos: Para la descripción de la composición de especies de la comunidad de hormigas se detalló la cantidad de especímenes y especies por subfamilia y género. La estimación de la riqueza de especies se analizó a diferentes escalas: a) la diversidad alfa o riqueza de especies en cada uno de los fragmentos de bosque; b) la diversidad beta, calculada con el índice de complementariedad de Colwell \& Coddington, (1994), se representó como un dendrograma para evaluar la similitud de los fragmentos en función de la riqueza de especies y; c) la diversidad gamma y representatividad del muestreo calculada mediante los estimadores no paramétricos Chaol y Jackknife1 representados como curvas de acumulación de especies (Chazdon, Colwell, Denslow \& Guariguata, 1998; Colwell \& Coddington, 1994).

Para realizar la comparación de la riqueza y abundancia de especies durante cada una de las temporadas climáticas se realizó una prueba de Wilcoxon pareada (prueba de U) comparando cada una de las unidades muestrales en la temporada seca y de lluvias. Para comparar la riqueza de especies en los ambientes de sabana, borde, bosque se realizó una prueba de ANOVA usando cada uno de los tres ambientes como un grupo. Con el fin de establecer la composición de especies en los ambientes de muestreo se realizó un Análisis de Escalamiento Multidimensional no Métrico (NMDS) usando la abundancia de especies por estaciones de muestreo en cada uno de los ambientes.

Para cada uno de los fragmentos de bosque se calculó el área (A) y perímetro (P) usando imágenes de satélite y se estimaron los índices de compactación (K) (Bosch, 1978) y dimensión fractal (DF) (Krummel, Gardner, Sugihara, O’Neill \& Coleman, 1987). Finalmente, para relacionar las especies con las variables del fragmento se llevó a cabo un Análisis de Correspondencia Canónica (ACC) usando los atributos del fragmento y las abundancias de especies dentro cada uno de los fragmentos de bosque. 


\section{RESULTADOS}

Se recolectaron e identificaron 30496 especímenes que pertenecían a 104 especies, 37 géneros y ocho subfamilias (Apéndice Digital). La subfamilia mejor representada fue Myrmicinae con 53 especies (51\%), seguida por Ponerinae con 15 (14.4\%), Formicinae $13(12.5 \%)$ y Dolichoderinae 12 (11.5\%). Las subfamilas Amblyoponinae, Dorylinae, Pseudomyrmicinae y Ectatomminae estuvieron representadas por menos de cinco especies cada una $(<4 \%$ del total $)$.

Respecto a la diversidad de géneros, la subfamilia Myrmicinae estuvo representada por 16 géneros (41\%), seguida por Ponerinae con siete $(17.9 \%)$, Dolichoderinae con cinco $(12.8 \%)$ y Formicinae con cuatro $(10.3 \%)$. Las cuatro subfamilias restantes estuvieron representadas por tres géneros o menos ( $>10 \%)$. Los géneros mejor representados fueron: Pheidole con 14 especies, seguido de Camponotus (nueve especies), Solenopsis (ocho especies) y Dolichoderus (siete especies). Los 33 géneros restantes estuvieron representados por cinco especies o menos.

La diversidad alfa, que representa el número de especies en cada una de las localidades, osciló entre 23 y 37 especies $(29.25 \pm 4.99)$. La mitad de los fragmentos presentaron riquezas mayores 30 especies (Tabla 1). Por otro lado, la diversidad beta representada como un dendrograma generó la formación de dos grandes grupos, aparentemente en respuesta a la distancia de los fragmentos al casco urbano de la ciudad de Arauca (Fig. 1). La diversidad gamma indicó que en la riqueza de hormigas esperada podría oscilar entre 107 (Chao 1) y 119 (Jackknife 1) especies respectivamente. Así, las 104 especies recolectadas representan entre el 87.5 y $97.2 \%$ de las especies de hormigas epígeas presentes en la zona.

Respecto a la riqueza de especies en los ambientes sabana-borde-bosque, los tres compartieron 35 especies; mientras que 25 especies fueron exclusivas del bosque; 13 fueron del borde y nueve de la sabana. El resultado de la prueba de ANOVA por ambientes mostró diferencias en la riqueza de especies entre cada uno de los ambientes muestreados (g.l. $=53, \mathrm{~N}=$ $56, \mathrm{~F}=12.96, \mathrm{P}<0.0001$ ), siendo la riqueza de especies de la sabana menor que la del bosque y el borde (Tukey: sabana-bosque $\mathrm{P}<0.0001$, sabana-borde: $\mathrm{P}<0.004)$.

El NMDS realizado para evaluar la composición de especies con respecto a cada uno de los ambientes mostró un ordenamiento donde se distinguen dos grupos, uno que corresponde a ambiente de sabana y el otro al borde y bosque agrupados (Estrés $=0.23$ ) (Fig. 2). La ordenación evidencia que especies como $\mathrm{Bra}$ chymyrmex sp1, Pheidole sp8, Pheidole sp9, Pheidole sp13, Pheidole sp14, Pseudomyrmex sp4 y Solenopsis sp4 tienen una alta afinidad

TABLA 1

Géneros y especies de hormigas recolectados en cada fragmento de bosque y sabana inundable del municipio de Arauca

TABLE 1

Genera and species of ants collected in each forest fragment and floodplain of Arauca municipality. Acronyms, area and perimeter for each fragment are shown

\begin{tabular}{lccccc}
\multicolumn{1}{c}{ Localidad } & Acrónimo & Géneros & Especies & Área (ha) & Perímetro (m) \\
Finca Los Laureles & LL & 23 & 37 & 148 & 9410.4 \\
Finca Las Mercedes del Llano sitio I & LMI & 22 & 33 & 8.7 & 2323 \\
Granja Universidad Nacional & G_UN & 23 & 32 & 1.5 & 707.1 \\
Finca Piedraca & FP & 19 & 31 & 21 & 2087 \\
Finca Mata de Gallina & FM & 19 & 29 & 47 & 3530.2 \\
Sendero Ecológico & S_ECO & 17 & 26 & 0.51 & 348.2 \\
Finca El Desastre & ED & 13 & 23 & 220 & 10524.6 \\
Finca Las Mercedes del Llano sitio II & LMII & 16 & 23 & 8 & 1704.3 \\
\hline
\end{tabular}




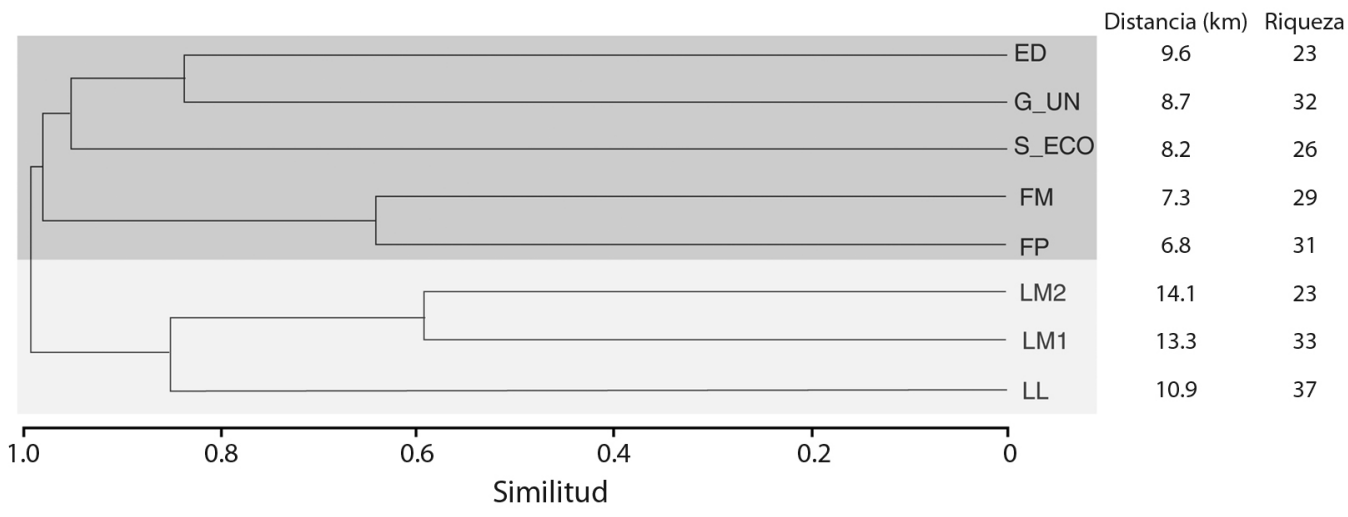

Fig. 1. Dendrograma de similitud usando el índice de complementariedad entre las ubicaciones de muestreo de hormigas en la sabana inundable del municipio de Arauca. El rectángulo inferior delimita el grupo formado por tres localidades. El segundo grupo formado por cinco localidades. Detalle de los acrónimos Tabla 1. Los valores a la derecha del dendrograma indican la distancia en kilómetros al centro urbano del municipio de Arauca y la riqueza de especies.

Fig. 1. Similarity dendrogram using complementarity index among ant sampling locations in the floodplain of the municipality of Arauca. The lower rectangle includes a group of three localities. The other group includes five localities. For details on acronyms, see Table 1 . The values to the right of dendrogram indicate the distance in kilometers to the urban zone of Arauca municipality and the species richness.

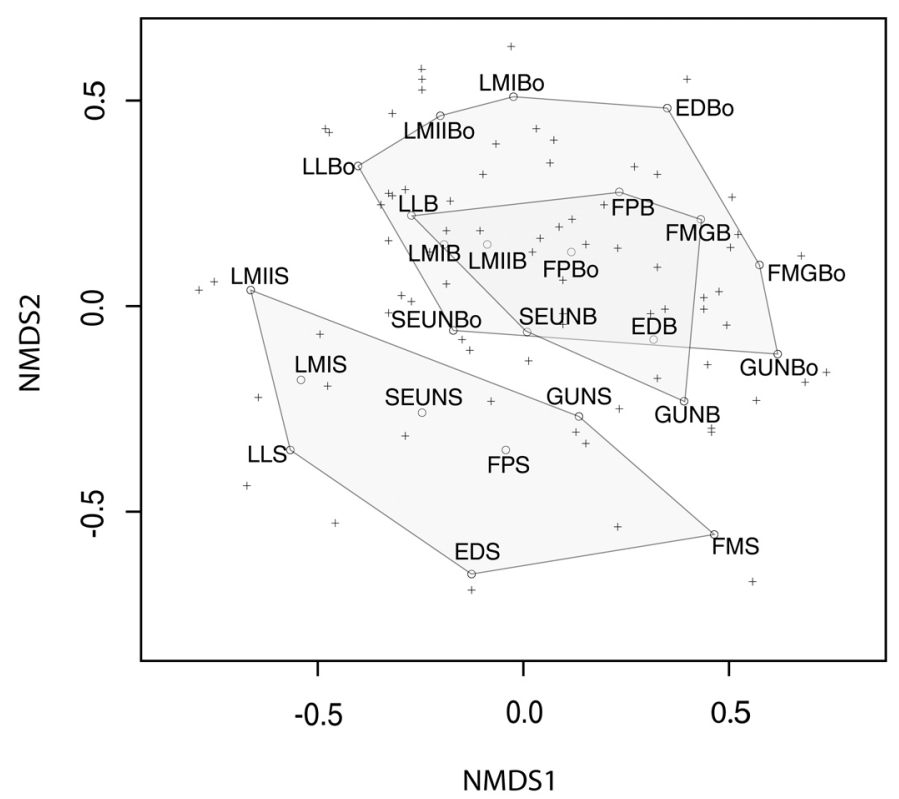

Fig. 2. Análisis de escalamiento multidimensional no métrico (NMDS) para la comunidad de hormigas en la sabana inundable del municipio de Arauca. Los polígonos representan los ambientes, los círculos representan cada una de las localidades de muestreo y las cruces representan las especies. Estrés 0.23.

Fig. 2. Non-metric Multidimensional Scaling (NMDS) Analysis for the ant community in the floodplain savanna of the municipality of Arauca. Polygons represent environments, circles represent each of the sampling locations and crosses represent species. Stress 0.23 . 
con el ambiente de sabana, mientras que en el bosque y borde se destacan las especies: Adelomyrmex sp1, Gnamptogenys regularis y Thaumatomyrmex cf. mutilatus que tienen afinidad tanto a hojarasca como a espacios abiertos y especies de los géneros Cephalotes, Dolichoderus, Carebara y Prionopelta que suelen ocupar ambientes de bosques y la hojarasca (Fernández, 2003).

En relación con la estacionalidad climática, la abundancia y riqueza de especies tuvieron comportamientos diferentes en cada una de las temporadas climáticas. La riqueza de especies durante las lluvias fue de 100 especies (cuatro especies exclusivas) y en la temporada seca de 89 especies (18 especies exclusivas). En el caso de la abundancia el comportamiento fue inverso, con una mayor abundancia de individuos en la temporada seca (19 691 individuos) con respecto a la temporada de lluvias (10 887 individuos). Sin embargo, esas diferencias no fueron significativas (riqueza: g.1. $=54, \mathrm{U}=$ 275.5; $\mathrm{N}=56 ; \mathrm{P}=0.07$, abundancia: g.l. $=54$; $\mathrm{U}=588, \mathrm{~N}=56, \mathrm{P}=0.6$ ).

El análisis correlación canónica para evaluar la relación entre las especies y los atributos de los fragmentos de bosque mostró una variación total explicada del $62.7 \%(\mathrm{P}=0.057, \alpha=$ $0.1)$ sugiriendo que la riqueza de especies en las ocho localidades está relacionada linealmente con las variables del paisaje área, perímetro, dimensión fractal y compactación (Fig. 3).

\section{DISCUSIÓN}

Los resultados de los estimadores no paramétricos de riqueza de especies revelaron que la eficiencia del muestreo estuvo entre el 87.5 y el $97.2 \%$ indicando que los métodos empleados fueron efectivos para documentar la riqueza de especies de hormigas epígeas en la comunidad. Las 104 especies de hormigas

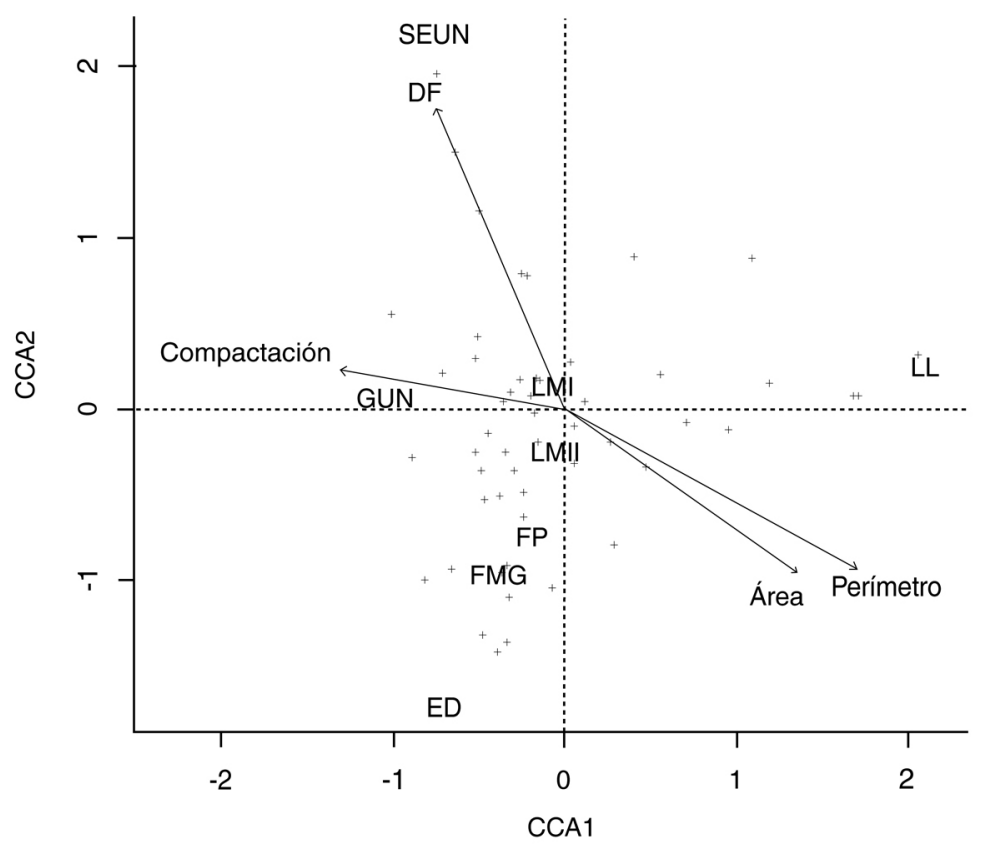

Fig. 3. Biplot basado en el análisis de correspondencia canónica de las variables del paisaje y las especies de hormigas en la sabana inundable del municipio de Arauca. Las cruces representan especies. Detalles de los acrónimos del fragmento ver Tabla 1 .

Fig. 3. Biplot based on Canonical Correspondence Analysis of landscape variables and ant species in the floodplain of the municipality of Arauca. The crosses represent species. For details on fragment acronyms see Table 1. 
recolectadas corresponderían a la mayor riqueza de los estudios disponibles para la región de la Orinoquia Colombiana, dado que Fernández y Schneider (1989) registraron 95 especies en la reserva La Macarena (departamento del Meta) y Sanabria, Lavelle y Fonte (2014) 91 en agroecosistemas, bosque y sabana en Puerto López y Puerto Gaitán en el Meta. Este resultado tiene que ver probablemente con tres factores: primero, la inclusión de los tres ambientes que predominan en esta región biogeográfica (sabana inundable, borde y bosque); segundo, la intensidad del muestreo que supera en número de horas trampa y de tiempo de recolecta manual a los trabajos antes referenciados y, tercero, la escala temporal que incluyo recolección de hormigas en las dos temporadas climáticas (lluvias y seca). Sin embargo, la riqueza de este estudio es menor en otras localidades de Colombia como el Cauca (113 especies Arenas-Clavijo \& Armbrecht, 2018) y en localidades de Antioquia y Risaralda (115 especies) (Armbrecht, Rivera \& Perfecto, 2005). En cuanto a la composición de especies se destacó la riqueza de la subfamilia Myrmicinae (53 especies), seguida de Ponerinae (15 especies) y Formicinae (13 especies). Esta composición es similar a otras localidades de la Orinoquia (Fernández \& Schneider, 1989; Pérez-Sánchez, Lattke \& Viloria, 2013; Sanabria et al., 2014) y es frecuente en ecosistemas del Neotrópico (Muñoz, Rousseau, Andrade-Silva, \& Delabie, 2017; Vicente, Ferreira, Santos, \& Prado, 2018)

Respecto a la diversidad beta, el dendrograma de similitud generó dos grupos de acuerdo con la similitud en la riqueza de especies el primer grupo mostró las tres localidades que se encuentran más alejadas del centro urbano de la ciudad de Arauca que presentan riquezas entre 23 y 37 especies; mientras que el segundo grupo lo constituían cinco localidades que se encuentran más cerca del centro urbano de la ciudad de Arauca y tuvieron riquezas entre 23 y 32 especies. Este resultado sugiere que la proximidad al casco urbano podría influenciar la composición de la comunidad de hormigas. El aumento de la riqueza de especies a la par con el aumento de la distancia al centro urbano de Arauca podría ser explicado por la hipótesis de heterogeneidad ambiental de Pianka (1966), la cual plantea que ambientes más heterogéneos proporcionan una mayor cantidad y diversidad de recursos que los ambientes simples, lo que resulta en una mayor diversidad de especies.

Esta hipótesis ha sido validada en hormigas en cultivos de café con manejo tradicional el café en Caldono (Cauca, Colombia) (Urrutia-Escobar \& Armbrecht 2013) así como también por factores como el aumento de la urbanización de ambientes naturales y rurales (Buczkowski \& Richmond, 2012; Lutinski, Lutinski, Lopes \& De Morais, 2014; SantosSilva, Vicente \& Feitosa, 2016). En Arauca, el comportamiento de la riqueza de hormigas en la sabana inundable de Arauca parece seguir este patrón en donde el casco urbano de Arauca actuaría como como centro de perturbación que "simplifica" la estructura de los ambientes cercanos lo que produce una disminución en la riqueza de especies. En el área de estudio, varios factores podrían estar incidiendo negativamente en la riqueza de hormigas en áreas próximas al casco urbano tales como la agricultura intensiva del cultivo de arroz y la deforestación asociada para incrementar el área cultivable, el uso de técnicas culturalmente arraigadas que tienen altos impactos ambientales como quemas para acondicionamiento de suelos y la fumigación aérea con insecticidas. Las perturbaciones antrópicas que ocurren en Arauca podrían validar la hipótesis de Pianka (1966) en el sistema estudiado, sin embargo, estudios adicionales son requeridos para corroborar el planteamiento que realizamos en este estudio.

En relación con la variación climática, está ampliamente documentado que la distribución, abundancia y riqueza de especies de muchos grupos de insectos pueden ser influenciadas por factores climáticos (Agudelo-Martínez, Gómez-Tapia \& Pérez-Buitrago, 2018; Wolda, 2003). En este estudio no se detectaron diferencias en la riqueza y abundancia de especies entre las temporadas de lluvias y sequía en la comunidad de hormigas, lo cual sugiere que la mayoría de las especies encontradas en la zona 
de estudio son residentes permanentes de los fragmentos de bosque y la sabana inundable; aunque en Brasil se han registrado diferencias en esas variables asociadas a factores climáticos cíclicos como inundaciones anuales (Majer \& Delabie, 1994; Soares et al., 2013; Vasconcelos, Vilhena, Facure, \& Albernaz, 2010).

En Arauca, el comportamiento de la comunidad de hormigas sugiere que las especies presentes en sabana poseen diferentes mecanismos para sobrellevar el periodo de inundación, en los que se pueden incluir por ejemplo el movimiento de las colonias de lugares inundables (i.e. la sabana) a los bordes o al interior de los fragmentos de bosque durante la temporada de lluvias (Adis, 1982; LeBrun, Moffett \& Holway, 2011; McGlynn, 2011) o movimientos verticales de las colonias a las partes altas en los lugares donde la microtopografía del terreno lo permita (Hakala, Seppä, \& Helanterä, 2019; Jacquemin, Roisin, \& Leponce, 2016; McGlynn, 2011).

El ANOVA para comparar la riqueza de especies por ambiente indicó que la sabana tuvo una riqueza menor con respecto al borde y el bosque. Este resultado coincide con la ordenación de las estaciones de muestreo de acuerdo al ambiente producto del NMDS (Fig. 3) en el que se evidencia que el ambiente de sabana es diferente de los ambientes de bosque y borde. Especies como Brachymyrmex sp1, Pheidole sp8, Pheidole sp9, Pheidole sp13, Pheidole sp14, Pseudomyrmex sp4 y Solenopsis sp4 son características del ambiente de sabana y serían las que determinan una diferencia con respecto a los ambientes de borde y bosque. Estos géneros son catalogados como generalistas en la explotación de recursos alimenticios (Silvestre, Brandão \& Silva, 2003).

Esta característica, conjugada con la fuerte estacionalidad, podría explicar su dominancia en el ambiente de sabana, puesto que durante la temporada seca estas especies tendrían una mayor eficiencia para explotar recursos alimenticios y de esta forma ser más comunes durante esta temporada Este comportamiento ha sido reportado en bosques primarios, secundarios y sistemas agroforestales en las regiones del
Pantanal y la Amazonía brasileña (Muñoz et al., 2017; Soares et al., 2013). Este mecanismo que involucra la capacidad de especies generalistas de explotar recursos alimenticios durante la temporada seca, podría contribuir a explicar porque existen diferencias en la riqueza de especies entre el ambiente de sabana inundable y los ambientes de borde y bosque documentados en este trabajo.

En cuanto al efecto de las variables del fragmento de bosque sobre la comunidad de hormigas, el análisis de correlación canónica indicó que el área, el perímetro, la dimensión fractal y la compactación influyen sobre la comunidad de hormigas, existiendo una relación directa entre el área, perímetro y compactación e inversa con la dimensión fractal. El efecto del tamaño del fragmento condiciona la abundancia siguiendo la teoría de biogeografía de islas (MacArthur \& Wilson, 2016) que plantea que las islas (en este caso fragmentos de bosque) de mayor tamaño pueden albergar mayor número de especies e individuos que las islas de menor tamaño y ha sido ampliamente documentado para múltiples taxones (Gillespie \& Roderick, 2002). Por otro lado, la relación inversa con la dimensión fractal sugiere que la desviación de los fragmentos de bosque con respecto a formas euclidianas regulares (i.e. círculos o cuadrados) afecta negativamente la riqueza de especies.

En este estudio los fragmentos de formas más irregulares tienen menor riqueza de especies producto del efecto de borde que puede llegar a ejercer la matriz sobre los fragmentos de bosque con mayor exposición a este. El efecto borde se ha definido como el cambio en la distribución de una variable (i.e. la riqueza o abundancia de especies) en la transición entre hábitats $\mathrm{y}$ ha sido documentado para muchos grupos taxonómicos de plantas y animales (Lidicker, 1999). En el caso de la familia Formicidae el efecto borde ha sido documentado en fragmentos de bosque primario y secundario en Brasil (Marques, Espiríto-Santo, Neves \& Schoereder, 2017; Sobrinho \& Schoereder, 2007) y fragmentos de bosque seco en Colombia (Lozano-Zambrano et al., 2009). En estos 
estudios, a pesar de no reportar una tendencia significativa del incremento de la riqueza de especies en el borde de los fragmentos, si se reconocen diferencias entre las riquezas de bordes y el interior de los fragmentos estudiados. En el caso de la sabana inundable en Arauca la riqueza de especies sigue esta misma tendencia lo que sugiere que existe una mayor susceptibilidad de los fragmentos pequeños a los efectos de borde, en donde es más probable encontrar especies de sabana y borde dentro de los fragmentos pequeños.

Las 104 especies de hormigas recolectadas en este estudio son representativas de los ecosistemas de sabana inundable y bosques asociados del norte de la Orinoquia colombiana, y se detectó una relación directa entre variables del fragmento de bosque como área, perímetro e índice de compactación, mientras que fue una relación inversa con la dimensión fractal. Esto evidencia como estos atributos de los fragmentos de bosque inciden directamente sobre la riqueza y composición de la comunidad de hormigas en esta región del país. Adicionalmente no se detectaron diferencias en la riqueza y abundancia de especies de hormigas entre las estaciones seca y lluviosa sugiriendo que las especies son residentes permanentes del área de estudio por lo que deben existir mecanismos que permitan a algunas especies de hormigas sobrellevar la estación lluviosa.

Declaración de ética: los autores declaran que todos están de acuerdo con esta publicación y que han hecho aportes que justifican su autoría; que no hay conflicto de interés de ningún tipo; y que han cumplido con todos los requisitos y procedimientos éticos y legales pertinentes. Todas las fuentes de financiamiento se detallan plena y claramente en la sección de agradecimientos. El respectivo documento legal firmado se encuentra en los archivos de la revista.

\section{AGRADECIMIENTOS}

A los profesores Benoit Jean Bernard Jahyny, Jacques Hubert Charles Delabie y a
William P. Mackay por su ayuda en la identificación y aportes bibliográficos. A Luis Ernesto Rodríguez, Juan Carlos Castañeda, Jairo Darío Piedrahita, Santiago Cuenza y la familia Garcés Bona, propietarios de los predios donde se recolectaron especímenes. Este trabajo fue financiado por "Convocatoria de Investigación para Financiar Pasantías, Trabajos de Grado en Pregrado o Posgrado en Temáticas Relacionadas con la Orinoquia 2013 II" de la Universidad Nacional de Colombia sede Orinoquia.

\section{RESUMEN}

Introducción: Las hormigas son uno de los grupos taxonómicos más abundantes y diversos, además su fidelidad ecológica les permite desempeñar roles indispensables para el funcionamiento adecuado de los ecosistemas terrestres. Objetivo: evaluar la dinámica estacional de la biodiversidad (riqueza y abundancia) de la comunidad de hormigas asociadas a fragmentos de bosque y la sabana inundable circundante (sabana-borde-bosque) en el municipio de Arauca (Colombia). Métodos: en un transecto lineal se recolectaron hormigas en siete puntos de muestreo usando trampas de caída, recolecta directa y saco mini-winkler para hojarasca en ocho fragmentos de bosque y la sabana circundante. Estimadores no paramétricos y el índice de complementariedad se utilizaron para describir la estructura de la comunidad de hormigas. Las estaciones climáticas (sequía y lluvias) y los ambientes (sabana inundable, borde y bosque) se compararon utilizando la riqueza y abundancia de especies. La relación de las especies de hormigas por cada punto de muestreo se evaluó con un análisis de escalamiento multidimensional no métrico (NMDS) y los atributos de los fragmentos de bosque se relacionaron con la riqueza de especies a través de un análisis de correspondencia canónica (CCA). Resultados: se recolectaron 30 496 especímenes correspondientes a 104 especies, 37 géneros y ocho subfamilias. Los géneros mejor representados fueron: Pheidole, Camponotus, Solenopsis y Dolichoderus. Los estimadores de riqueza no paramétricos mostraron que la comunidad de hormigas oscila entre 107 y 119 especies. No se detectaron diferencias en la riqueza y abundancia de especies respecto a las estaciones climáticas. La riqueza de especies fue mayor en el bosque respecto a la de la sabana y el borde. El resultado del ACC presentó una variación total explicada por el $62.7 \%$ que muestra la relación entre las especies y los atributos de los fragmentos de bosque. Conclusiones: no se detectaron diferencias en la riqueza y abundancia de especies entre las estaciones seca y lluviosa, sugiriendo que las especies son residentes permanentes del área de estudio, por lo que deben existir mecanismos que permitan a ciertas especies de hormigas sobrellevar la estación lluviosa. Las variables del fragmento área, perímetro y compactación presentan una relación directa con 
la riqueza de especies, mientras que la dimensión fractal tiene una relación inversa sobre la riqueza de especies que el fragmento alberga. Este trabajo amplía el conocimiento de las especies de hormigas presentes en el norte de la Orinoquia colombiana.

Palabras clave: paisaje; Formicidae; biodiversidad de hormigas; Arauca; patrón de riqueza de especies; estacionalidad.

\section{REFERENCIAS}

Adis, J. (1982). Eco-entomological observations from the Amazon: III how do the leafcutting ants of inundation forest survive flooding. Acta Amazonica, 12(4), 839.

Agosti, D., Majer, J.D., Alonso, L.E., \& Schultz, T.R. (2000). ANTS Standard methods for measuring and monitoring biodiversity. Biological Diversity Handbook Series, 233, 280. DOI: https://doi.org/10.1148/ radiol.2332031110

Agudelo-Martínez, J.C., Gómez-Tapia, E.M., \& PérezBuitrago, N. (2018). Dinámica temporal de la riqueza de especies y la abundancia de mariposas frugívoras (Lepidoptera: Nymphalidae) en la sabana inundable del municipio de Arauca (Colombia). Revista de La Academia Colombiana de Ciencias Exactas, Físicas y Naturales, 42(164), 246. DOI: https://doi. org/10.18257/raccefyn.672

Agudelo-Martínez, J.C., \& Pérez-Buitrago, N. (2015). Notas acerca de la distribución de Papilionidae (Lepidoptera: Papilionoidea) en el norte de la Orinoquia Colombiana. Boletín Cientifico. Centro de Museos. Museo de Historia Natural, 19(1), 203-214. DOI: https://doi.org/10.17151/bccm.2015.19.1.14

Andersen, A.N., Fisher, A., Hoffmann, B.D., Read, J.L., \& Richards, R. (2004). Use of terrestrial invertebrates for biodiversity monitoring in Australian rangelands, with particular reference to ants. Austral Ecology, 29(1), 87-92. DOI: https://doi. org/10.1111/j.1442-9993.2004.01362.x

Andersen, A.N., \& Majer, J.D. (2004). Ants show the way Down Under: invertebrates as bioindicators in land management. Frontiers in Ecology and the Environment, 2(6), 291-298. DOI: https://doi. org/10.1890/1540-9295(2004)002[0292:astwdu]2.0. co;2

Arbeláez-Cortés, E. (2013). Knowledge of Colombian biodiversity: Published and indexed. Biodiversity and Conservation, 22(12), 2875-2906. DOI: https://doi. org/10.1007/s10531-013-0560-y

Arenas-Clavijo, A., \& Armbrecht, I. (2018). Gremios y diversidad de hormigas (Hymenoptera: formicidae) en tres usos del suelo de un paisaje cafetero del Cauca-colombia. Revista de Biologia Tropical, 66(1), 48-57. DOI: https://doi.org/10.15517/rbt.v66i1.30269
Armbrecht, I., Rivera, L., \& Perfecto, I. (2005). Reduced Diversity and Complexity in the Leaf-LitterAntAssemblage of Colombian Coffee Plantations Reducción en la Diversidad y Complejidad del Ensamble de Hormigas de la Hojarasca en Plantaciones de Café Colombianas. Conservation Biology, 19(3), 897-907. DOI: https://doi.org/10.1111/j.1523-1739.2005.00062.x

Baccaro, F.B., Rocha, I.F., Aguila, B.E.G., Schietti, J., \& Emilio, T. (2013). Changes in Ground-dwelling Ant Functional Diversity are Correlated with Water-Table Level in an Amazonian Terra Firme Forest. Biotropica, 45(6), 755-763.

Bolton, B. (1994). Identification guide to the ant genera of the world. Cambridge, USA: Harvard University Press.

Bosch, W. (1978). A procedure for quantifying certain geornorphological features. Geographical Analysis, $X(3), 241-247$.

Brown, W.L.J. (1976). Contributions toward a reclassification of the Formicidae. Part VI. Ponerinae, tribe Ponerini, subtribe Odontomachiti. Section A. Introduction, subtribal characters. Genus Odontomachus. Studia Entomologica, 19, 67-171.

Brown, W.L.J. (1978). Contributions toward a reclassification of the Formicidae. Part VI. Ponerinae, Tribe Ponerini, Subtribe Odontomachiti. Section B. Genus Anochetus and bibliography. Studia Entomologica, 19, 549-638.

Buczkowski, G., \& Richmond, D.S. (2012). The effect of urbanization on ant abundance and diversity: A temporal examination of factors affecting biodiversity. PLoS ONE, 7(8), 22-25. DOI: https://doi. org/10.1371/journal.pone.0041729

Chazdon, R.L., Colwell, K.R., Denslow, S.J., \& Guariguata, R.M. (1998). Statistical methods for estimating species richness of woody regeneration in primary and secondary rain forests of NE Costa Rica. In J.A. Dallmeier, \& F. Comiskey (Eds.), Forest biodiversity research, monitoring and modeling: Conceptual background and Old World case studies (pp. 285-309). Washington D.C., USA: UNESCO Paris \& The Parthenon Publishing Group.

Colwell, R.K., \& Coddington, J.A. (1994). Estimating terrestrial biodiversity through extrapolation. Philosophical Transactions of the Royal Society of London. Series B, Biological Sciences, 345(1311), 101-118. DOI: https://doi.org/10.1098/rstb.1994.0091

Correa, H.D., Ruiz, S.L., \& Arévalo, L.M. (Eds.). (2005). Plan De Acción En Biodiversidad De La Cuenca Del Orinoco Colombia. Bogotá, Colombia: Corporinoquia, Cormacarena, I.A.v.H, Unitrópico, Fundación Omacha, Fundación Horizonte Verde, Universidad Javeriana, Unillanos, WWF - Colombia, GTZ. 
Falcão, J.C.F., Dáttilo, W., \& Izzo, T.J. (2015). Forest Ecology and Management Efficiency of different planted forests in recovering biodiversity and ecological interactions in Brazilian Amazon. Forest Ecology and Management Journal, 339, 105-111. DOI: https://doi. org/10.1016/j.foreco.2014.12.007

Fernández, F. (2003). Introducción a las hormigas de la región Neotropical. Bogotá D. C., Colombia: Instituto de Investigación de Recursos Biológicos Alexander von Humboldt.

Fernández, F., \& Schneider, L. (1989). Reconocimiento de hormigas en la Reserva La Macarena. Revista Colombiana de Entomología, 15(1), 38-44.

Folgarati, P.F. (1998). Ant biodiversity and its realtionship to ecosystem function: a review. Biodiversity and Conservation, 7, 1221-1244.

Gerlach, J., Samways, M., \& Pryke, J. (2013). Terrestrial invertebrates as bioindicators: An overview of available taxonomic groups. Journal of Insect Conservation, 17(4), 831-850. DOI: https://doi.org/10.1007/ s10841-013-9565-9

Gillespie, R.G., \& Roderick, G.K. (2002). Arthropods on islands: Colonization, speciation, and conservation. Annual Review of Entomology, 47, 595-632. DOI: https://doi.org/10.1146/annurev. ento.47.091201.145244

Gropp, R.E. (2006). Are University Natural Science Collections Going Extinct? BioScience, 53(6), 550. DOI: https://doi. org/10.1641/0006-3568(2003)053[0550:aunscg]2.0. $\operatorname{co} ; 2$

Hakala, S.M., Seppä, P., \& Helanterä, H. (2019). Evolution of dispersal in ants (Hymenoptera: Formicidae): a review on the dispersal strategies of sessile superorganisms. Myrmecological News, 29, 35-55. DOI: https://doi.org/10.25849/myrmecol.news

Jacquemin, J., Roisin, Y., \& Leponce, M. (2016). Spatiotemporal variation in ant (Hymenoptera: Formicidae) communities in leaf-litter and soil layers in a premontane tropical forest. Myrmecological News, 22, 129-139.

Jiménez, E., Fernández, F., Arias, T.M., \& Lozano-Zambrano, F.H. (Eds.). (2007). Sistemática, biogeografia y conservación de las hormigas cazadoras de Colombia. Bogotá D. C., Colombia: Instituto de Investigación de Recursos Biológicos Alexander von Humboldt.

Krummel, J.R., Gardner, R.H., Sugihara, G., O’Neill, R.V., \& Coleman, P.R. (1987). Landscape Patterns in a Disturbed Environment. Oikos, 48(3), 321. DOI: https:// doi.org/10.2307/3565520

LeBrun, E.G., Moffett, M., \& Holway, D.A. (2011). Convergent evolution of levee building behavior among distantly related ant species in a floodplain ant assemblage. Insectes Sociaux, 58(2), 263-269. DOI: https:// doi.org/10.1007/s00040-011-0151-4

Lidicker, W.Z. (1999). Responses of mammals to habitat edges: An overview. Landscape Ecology, 14(4), 333343.DOI: https://doi.org/10.1023/A:1008056817939

Lozano-Zambrano, F.H., Ulloa-Chacón, P., \& Armbrecht, I. (2009). Hormigas: relaciones especies-área en fragmentos de bosque seco tropical. Neotropical Entomology, 38(1), 44-54. DOI: https://doi.org/10.1590/ S1519-566X2009000100004

Lutinski, J.A., Lutinski, C.J., Lopes, B.C., \& De Morais, A.B.B. (2014). Estrutura da comunidade de formigas (Hymenoptera: Formicidae) em quatro ambientes com diferentes níveis de perturbação antrópica. Ecologia Austral, 24(2), 229-237.

MacArthur, R.H., \& Wilson, E.O. (2016). The Theory of Island Biogeography. New Jersey, United States: Princeton University Press. DOI: https://doi. org/10.1515/9781400881376

Mackay, W., \& Mackay, E. (2010). The systematics and biology of the New World ants of the genus Pachycondyla: Formicidae. Lewiston, N.Y, United States: Edwin Mellen Press.

Majer, J.D. (1983). Ants: Bio-indicators of minesite rehabilitation, land-use, and land conservation. Environmental Management, 7(4), 375-383. DOI: https://doi. org/10.1007/BF01866920

Majer, J.D., \& Delabie, J.H.C. (1994). Comparison of the ant communities of annually inundated and terra firme forests at Trombetas in the Brazilian Amazon. Insectes Sociaux, 359, 343-359.

Marques, T., Espiríto-Santo, M.M., Neves, F.S., \& Schoereder, J.H. (2017). Ant assemblage structure in a secondary tropical dry forest: The role of ecological succession and seasonality. Sociobiology, 64(3), 261-275. DOI: https://doi.org/10.13102/sociobiology. v64i3.1276

Mcgeoch, M.A. (2014). The selection, testing and application of terrestrial insects as bioindicators. Biologial Reviews, 73, 181-201.

McGlynn, T.P. (2011). The Ecology of Nest Movement in Social Insects. Annual Review of Entomology, 57(1), 291-308. DOI: https://doi.org/10.1146/ annurev-ento-120710-100708

Minorta-Cely, V., \& Rangel-Ch, J.O. (2014). La riqueza y diversidad de las plantas con flores de la orinoquia Colombiana. In J.O. Rangel-Ch (Ed.), Colombia, diversidad biótica XIV. La región de la Orinoquia de Colombia (pp. 533-608). Bogotá, Colombia: Instituto de Ciencias Naturales, Universidad Nacional de Colombia. 
Muñoz, G.J.A., Rousseau, G.X., Andrade-Silva, J., \& Delabie, J.H.C. (2017). Taxones superiores de hormigas como sustitutos de la riqueza de especies, en una cronosecuencia de bosques secundarios, bosque primario y sistemas agroforestales en la Amazonía Oriental, Brasil. Revista de Biología Tropical, 65(1), 279-291. DOI: https://doi.org/10.15517/rbt.v65i1.23526

Myers, N. (1996). The biodiversity crisis and the future of evolution. Environmentalist, 16(1), 37-47. DOI: https://doi.org/10.1007/BF01325613

Palacio, E.E., \& Fernández, F. (2003). Clave para las subfamilias y géneros. In F. Fernández (Ed.), Introducción a las hormigas de la región Neotropical (Vol. 19, pp. 233-260). Bogotá, Colombia: Instituto de Investigación de Recursos Biológicos Alexander von Humboldt.

Pérez-Sánchez, A.J., Lattke, J.E., \& Viloria, A.L. (2013). Patterns of Ant (Hymenoptera: Formicidae) Richness and Relative Abundance along an Aridity Gradient in Western Venezuela. Neotropical Entomology, 42(2), 128-136. DOI: https://doi.org/10.1007/ s13744-012-0096-y

Pianka, E.R. (1966). Convexity, Desert Lizards, and Spatial Heterogeneity. Ecology, 47(6), 1055-1059.

Rangel-Ch, J.O. (2005). La biodiversidad de Colombia. Palmpsesto Facultad De Ciencias Humanas - Universidad Nacional De Colombia, 39(151), 292-304.

Rippstein, G., Amézquita, E., Escobar, G., \& Grollier, C. (2001). Condiciones Naturales de la Sabana. En G. Rippstein, G. Escobar, \& F. Motta (Eds.), Agroecología y biodiversidad de las sabanas en los llanos orientales de Colombia (pp. 1-21). Cali, Colombia: Centro Internacional de Agricultura Tropical.

Sanabria, C., Lavelle, P., \& Fonte, S.J. (2014). Ants as indicators of soil-based ecosystem services in agroecosystems of the Colombian Llanos. Applied Soil Ecology, 84, 24-30. DOI: https://doi.org/10.1016/j. apsoil.2014.07.001

Santos-Silva, L., Vicente, R.E., \& Feitosa, R.M. (2016). Ant species (Hymenoptera, Formicidae) of forest fragments and urban areas in a Meridional Amazonian landscape. Check List, 12(3), 1885. DOI: https:// doi.org/10.15560/12.3.1885

Silvestre, R., Brandão, C.R.F., \& Silva, R.R. (2003). Introducción a las hormigas de la región Neotropical. In F. Fernández (Ed.), Introducción a las hormigas de la región Neotropical (pp. 113-148). Bogotá, Colombia: Instituto de Investigación de Recursos Biologicos Alexander von Humboldt.
Soares, S. de A., Suarez, Y.R., Fernandes, W.D., Tenório, P.M.S., Delabie, J.H.C., \& Antonialli-Junior, W.F. (2013). Temporal variation in the composition of ant assemblages (Hymenoptera, Formicidae) on trees in the Pantanal floodplain, Mato Grosso do Sul, Brazil. Revista Brasileira de Entomologia, 57(1), 84-90. DOI: https://doi.org/10.1590/ s0085-56262013000100013

Sobrinho, T.G., \& Schoereder, J.H. (2007). Edge and shape effects on ant (Hymenoptera: Formicidae) species richness and composition in forest fragments. Biodiversity and Conservation, 16(5), 1459-1470. DOI: https://doi.org/10.1007/s10531-006-9011-3

Solari, S., Muñoz-Saba, Y., Rodríguez-Mahecha, J., Defler, T., Ramírez-Chaves, H., \& Trujillo, F. (2013). De los mamíferos de Colombia. Mastozoología Neotropical, 20(2), 301-365.

Underwood, E.C., \& Fisher, B.L. (2006). The role of ants in conservation monitoring: If, when, and how. Biological Conservation, 132(2), 166-182. DOI: https:// doi.org/10.1016/j.biocon.2006.03.022

Urrutia-Escobar, M.X., \& Armbrecht, I. (2013). Effect of Two Agroecological Management Strategies on Ant (Hymenoptera: Formicidae) Diversity on Coffee Plantations in Southwestern Colombia. Environmental Entomology, 42(2), 194-203. DOI: https://doi. org/10.1603/EN11084

Vanegas, M.A.B. (2010). Efecto de la complejidad del hábitat en la composición de la comunidad de hormigas en bosques premontanos en el área de influencia de la central hidroeléctrica "Porce II" (Tesis de Maestría). Universidad Nacional de Colombia, Medellín, Colombia.

Vasconcelos, H.L., Vilhena, M.S., Facure, K.G., \& Albernaz, A.L.K.M. (2010). Patterns of ant species diversity and turnover across $2000 \mathrm{~km}$ of Amazonian floodplain forest. Journal of Biogeography, 37, 432-440. DOI: https://doi.org/10.1111/j.1365-2699.2009.02230.x

Vicente, R.E., Ferreira, A.C., Conceição, R., \& Prado, L.P. (2016). Ants ( Hymenoptera: Formicidae) from an Amazonian fragmented landscape, Juara , Mato Grosso, Brazil, with new records of ant species. Papéis Avulsos de Zoologia, 58, 0-4.

Wike, L.D., Martin, F.D., Paller, M.H., \& Nelson, E.A. (2010). Impact of Forest Seral Stage on use of Ant Communities for Rapid Assessment of Terrestrial Ecosystem Health. Journal of Insect Science, 10(77), 1-16. DOI: https://doi.org/10.1673/031.010.7701

Wolda, H. (2003). Insect Seasonality: Why? Annual Review of Ecology and Systematics, 19(1), 1-18. DOI: https:// doi.org/10.1146/annurev.es.19.110188.000245 